\title{
TIDAL LIMITS TO PLANETARY HABITABILITY
}

\author{
Rory Barnes $^{1,2}$, Brian JACKSON ${ }^{3}$, Richard GreEnberg ${ }^{3}$, And SEAN N. RAYMOnd ${ }^{2,4}$ \\ ${ }^{1}$ Department of Astronomy, University of Washington, Seattle, WA 98195-1580, USA \\ 2 Virtual Planetary Laboratory, USA \\ ${ }^{3}$ Lunar and Planetary Laboratory, University of Arizona, Tucson, AZ 85721, USA \\ ${ }^{4}$ Center for Astrophysics and Space Astronomy, University of Colorado, UCB 389, Boulder, CO 80309-0389, USA \\ Received 2009 April 30; accepted 2009 June 8; published 2009 June 30
}

\begin{abstract}
The habitable zones (HZs) of main-sequence stars have traditionally been defined as the range of orbits that intercept the appropriate amount of stellar flux to permit surface water on a planet. Terrestrial exoplanets discovered to orbit $\mathrm{M}$ stars in these zones, which are close-in due to decreased stellar luminosity, may also undergo significant tidal heating. Tidal heating may span a wide range for terrestrial exoplanets and may significantly affect conditions near the surface. For example, if heating rates on an exoplanet are near or greater than that on Io (where tides drive volcanism that resurfaces the planet at least every $1 \mathrm{Myr}$ ) and produce similar surface conditions, then the development of life seems unlikely. On the other hand, if the tidal heating rate is less than the minimum to initiate plate tectonics, then $\mathrm{CO}_{2}$ may not be recycled through subduction, leading to a runaway greenhouse that sterilizes the planet. These two cases represent potential boundaries to habitability and are presented along with the range of the traditional $\mathrm{HZ}$ for main-sequence, low-mass stars. We propose a revised HZ that incorporates both stellar insolation and tidal heating. We apply these criteria to GJ $581 \mathrm{~d}$ and find that it is in the traditional HZ, but its tidal heating alone may be insufficient for plate tectonics.
\end{abstract}

Key words: astrobiology - planetary systems - stars: individual (GJ 581) - stars: low-mass, brown dwarfs

\section{INTRODUCTION}

The discovery of extrasolar planets has made detecting and recognizing life-bearing planets outside the solar system a real possibility. Considerations of stellar radiation and climate led to the definition of a "habitable zone" (HZ) as a region around a star in which a planet with an Earth-like atmosphere could support liquid water on its surface (Kasting et al. 1993; Selsis et al. 2007; Spiegel et al. 2009). Close to the star, the surface temperature is too hot for liquid water; far from the star it is too cold; but in between is a range of orbits for which liquid water is stable on the surface. This definition predicts that habitable orbits lie closer $(\sim 0.1 \mathrm{AU})$ to low-mass stars than to Sun-like stars because low-mass stars are less luminous. We call this range of orbits the "insolation habitable zone" (IHZ).

Also of critical importance for determining habitability is the maximum mass that does not accrete a significant hydrogen-rich envelope. Numerous investigations have modeled this cutoff, and values range from 0.1 to 16 Earth masses $\left(M_{E}\right.$; Pollack et al. 1996; Ikoma et al. 2001; Lissauer et al. 2009). For the expected conditions of most protoplanetary disks, these results suggest that $10 M_{E}$ is a reasonable approximation for this critical mass, and hence, as is now standard, we assume that habitable planets must be less massive than this value.

Transit and radial velocity surveys are most sensitive to planets in small orbits $(\lesssim 0.5 \mathrm{AU})$, and hence are unlikely to find terrestrial planets in the IHZs of solar-mass stars. However, terrestrial-scale planets are readily detectable in the IHZs of low-mass stars (Mayor et al. 2009). Radial velocity surveys of $\mathrm{M}$ stars are complicated by the presence of stellar activity (West et al. 2004), which can induce surface fluctuations that erase detectable signals of terrestrial-scale planets. Nevertheless, the first plausibly terrestrial-sized exoplanet in the IHZ has just been discovered (GJ $581 \mathrm{~d}, \geqslant 7.1 M_{E}$; Mayor et al. 2009). Analogously, transit detections are hampered by starspots, which are more frequent and larger on $\mathrm{M}$ stars than on larger stars. Nonetheless, transit surveys may be the most effective method for discovering terrestrial exoplanets due to their ability to break the mass-inclination degeneracy of radial velocity data, and measure the planetary radius directly. With combined transit and radial velocity data, the unambiguous detection of a terrestrial planet in the IHZ is feasible in the immediate future. Not surprisingly, surveys such as "MEarth" have begun to scan M stars for transits (Nutzman \& Charbonneau 2008).

However, planets within a few tenths of an AU from M stars are in a very different environment than anywhere in the IHZ of our solar system: they may be hit with solar flares, bathed in X-rays due to increased stellar activity, and/or subjected to strong tidal effects. The first two are certainly concerns for the success of biology, although they were neglected in the original IHZ definition. Significant research has since explored these issues (e.g., Lammer et al. 2007; Segura et al. 2009; J. Zendejas et al. 2009, in preparation). Here we focus on the third issue and show that tides may place important constraints on planetary habitability.

For example, the recently announced $2 M_{E}$ planet GJ 581 e (Mayor et al. 2009) may experience intense tidal heating. Mayor et al. showed that this planet's eccentricity occasionally reaches values of 0.1. Applying common models of tidal heating (e.g., Peale et al. 1979; Jackson et al. 2008a, 2008b; Barnes et al. 2009), and assuming the planet to be terrestrial-like, GJ $581 \mathrm{e}$ could have 2 orders of magnitude more tidal heating than Jupiter's volcanic satellite Io! Although this planet is not in the IHZ, similar heating rates on planets in the IHZ are unlikely to develop life (Jackson et al. 2008a).

However, only in some cases will the heating be greater than Io's; in others it may lead to geophysical processes (e.g., plate tectonics) that maintain long-term climate stability, increasing potential habitability (Williams et al. 1997). Jackson et al. (2008a) showed that tidal heating in the center of the IHZ could be substantial for a few illustrative cases. Here we identify the boundaries of this "tidal HZ" (THZ) for a range 
of stellar and planetary masses, $M_{*}$, and $M_{p}$, respectively, as well as semimajor axis $a$ and orbital eccentricity $e$. This definition excludes radiogenic sources of heat, which dominate the heating on the Earth. Estimating radiogenic heating rates on exoplanets seems a daunting task as it depends sensitively on the composition of the planet (itself a result of many different formation pathways; Raymond et al. 2008), the age, and the internal structure of the planet. Therefore, bear in mind that the heating rates we present here are in addition to any radiogenic heating.

We define a new HZ to take into account tidal heat: planetary habitability requires liquid water on the surface and enough internal heat to drive plate tectonics, but not so much as to cause Io-like volcanism. Combined with the other restriction, these additional requirements significantly reduce the range of detectable habitable environments around low-mass stars. In Section 2, we describe the IHZ and THZ. In Section 3, we combine these models to introduce the "insolation-tidal HZ" (ITHZ) and define it for the cases of 1 and $10 M_{E}$ planets. In Section 4, we discuss the implications, and suggest directions for future research.

\section{TIDAL AND ATMOSPHERIC MODELS}

\subsection{The Insolation Habitable Zone}

Kasting et al. (1993) suggested that planetary habitability required liquid water to be stable on a planetary surface. They made simple assumptions about the atmosphere, and the planetary orbit (e.g., it was circular). Selsis et al. (2007) included the effects of cloud cover, and applied an updated version of the Kasting et al. model to the GJ 581 system (Udry et al. 2007). However, many of the known exoplanets are on eccentric orbits (Butler et al. 2006), suggesting rocky exoplanets will be also. Hence, the effect of eccentricity may be important when assessing habitability. Williams \& Pollard (2002) pointed out that planets on eccentric orbits tend to have surface temperatures that reflect the orbit-averaged stellar flux.

Barnes et al. (2008) modified the Selsis et al. (2007) model to reflect the Williams \& Pollard (2002) results in order to define an "eccentric HZ." Here we adopt the Barnes et al. definition of the IHZ, which requires a planet's semimajor axis to lie in the range $l_{\text {in }}<a<l_{\text {out }}$, where $l_{\text {in }}$ and $l_{\text {out }}$ are the inner and outer edge of the IHZ, respectively. As in Barnes et al., we assume all planets have $50 \%$ cloud cover (see Selsis et al. 2007).

\subsection{The Tidal Habitable Zone}

The exact mechanisms of tidal dissipation are poorly understood, but several quantitative models have been suggested (e.g., Goldreich \& Soter 1966; Hut 1981; Levrard et al. 2007; Ferraz-Mello et al. 2008; Efroimsky \& Lainey 2007; Wisdom 2008). A conventional model quantifies the tidal heating of a body as

$$
H=\frac{63}{4} \frac{\left(G M_{*}\right)^{3 / 2} M_{*} R_{p}^{5}}{Q_{p}^{\prime}} a^{-15 / 2} e^{2},
$$

where $G$ is the gravitational constant, $R_{p}$ is the planetary radius, and $Q_{p}^{\prime}$ is the "tidal dissipation function" which encapsulates the physical response of the body to tides, including the Love number (Peale et al. 1979; Jackson et al. 2008b). Note that this model may break down for large $e$. With the exception of $Q_{p}^{\prime}$, all these quantities can be measured for exoplanets. However, in lieu of actual mass and radius measurements we use the scaling relationship $R_{p} \propto M_{p}^{0.27}$ (Sotin et al. 2007).
In order to assess the surface effects of tidal heating on a potential biosphere, consider the heating flux, $h=H / 4 \pi R_{p}^{2}$, through the planetary surface. On Io, $h=2 \mathrm{~W} \mathrm{~m}^{-2}$ (from tidal heating; McEwen et al. 2004), resulting in intense global volcanism and a lithosphere recycling timescale of 142-3.6 $\times$ $10^{5}$ years (Blaney et al. 1995; McEwen et al. 2004). Such rapid resurfacing on an exoplanet probably precludes the development of a biosphere, hence we will assume that heating rates larger than $h_{\text {max }} \equiv 2 \mathrm{~W} \mathrm{~m}^{-2}$ result in uninhabitable planets.

Internal heating can also drive plate tectonics. This phenomenon may enable planetary habitability because it drives the carbonate-silicate cycle, stabilizing atmospheric temperatures and $\mathrm{CO}_{2}$ levels on timescales of hundreds of millions of years. Although the processes that drive plate tectonics are not fully understood on the Earth (Walker et al. 1981; Regenauer-Lieb et al. 2001), an adequate internal heat source is essential. Theoretical studies of Martian geophysics suggest tectonic activity ceased when the radiogenic heating flux $h_{\text {rad }}$ dropped below $0.04 \mathrm{~W} \mathrm{~m}^{-2}$ (Williams et al. 1997). We will therefore assume the planets have Earth-like weathering and that heating rates smaller than $h_{\text {min }} \equiv 0.04 \mathrm{~W} \mathrm{~m}^{-2}$ result in uninhabitable planets.

On Earth, the heating comes from the radioactive decay of $\mathrm{U}$ and $\mathrm{K}$ and produces a heat flux of $h_{\mathrm{rad} \oplus}=0.08 \mathrm{~W} \mathrm{~m}^{-2}$ (Davies 1999). In the case of exoplanets, the radiogenic heating rates are unknown, but they might well be inadequate. Planets significantly smaller than the Earth, which may be common around M stars (Raymond et al. 2007b), may be especially susceptible to insufficient heating (the radiogenic heat flux scales as the ratio of volume to area). In many cases, tidal heating probably dominates (Jackson et al. 2008a, 2008b), and may be directly estimated from the mass and orbit of the planet. Such a calculation helps assess a planet's internal heating and accompanying geophysical processes.

The above examples depend critically on the internal structure and composition of the body in question. Without this information, objects in the solar system serve as the best guide for hypothesizing the internal structure and dynamics of exoplanets. Hence, while explicitly acknowledging the wide range of planets likely to be discovered, we consider the above definitions of $h_{\min }$ and $h_{\max }$ to be the limits of habitability.

Therefore, we define the THZ to be the region around a star for which $h_{\min }<h<h_{\max }$ (where $h$ only represents the tidal heating), in this case, enough heat for plate tectonics, but not so much as to result in extremely rapid resurfacing. As with the IHZ, this definition makes critical assumptions about the planetary properties, specifically the planetary $Q_{p}^{\prime}$ value. We assume $Q_{p}^{\prime}=500$, consistent with the values of most terrestriallike bodies in the solar system (Dickey et al. 1994; Yoder 1995; Mardling \& Lin 2002).

\section{THE INSOLATION-TIDAL HABITABLE ZONE}

Habitable conditions may be present on a planet if it lies in both the IHZ and the THZ. In other words, $l_{\text {in }}<a<l_{\text {out }}$ and $h_{\min }<h<h_{\max }$. When both these requirements are met, a planet is in the ITHZ. In this section, we present examples of how this refinement modifies our view of the habitability of planets orbiting main-sequence stars.

Figure 1 shows the ITHZ as a function of $M_{*}$ and $a$ for a $10 M_{E}$ planet, the most detectable terrestrial-like exoplanet. The blue shading represents the IHZ (see Section 2.1); the yellow shading represents the THZ (see Section 2.2). For the 


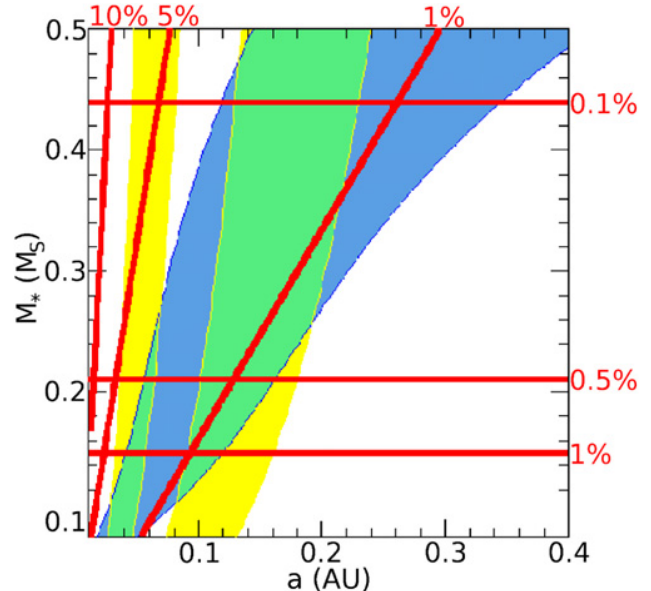

Figure 1. Boundaries of habitability for a $10 M_{E}$ terrestrial planet, and associated transit detectability levels. The blue region represents the IHZ boundaries for a planet with 50\% cloud cover (the boundaries change negligibly for $e<0.5$ ) The two yellow strips correspond to planets with favorable amounts of tidal heating if $e=0.01$ (left strip) or $e=0.5$ (right strip). Horizontal red lines correspond to different transit depths (with the value of each line to the right). Diagonal red lines represent geometric transit probability (with the value of each line at the top).

latter, two cases are shown: $e=0.01$ (left strip) and $e=0.5$ (right strip). The intersections of the IHZ and the THZ are shaded green and are two ITHZs. Apparently by chance, the two habitability requirements significantly overlap in these and many other plausible cases, but the range of habitable orbits is smaller when considering both tidal and insolation effects.

In order to assess the likelihood of finding bodies in the ITHZ, we show transit detection probabilities as the red lines in Figure 1. The horizontal ones are geometric transit probabilities (calculated from Barnes 2007), and the diagonal ones represent the percentage of starlight blocked during transit (the "transit depth," the ratio of the cross-sectional areas of the planet and star). Geometric probabilities increase with decreasing $a$, but transit depths are independent of $a$. Transit depths of $\sim 0.5 \%$ have been detected from the ground (e.g., Winn et al. 2008). Transiting terrestrial-scale planets are most likely to be observed in the lower left of this figure, and hence may experience significant tidal heating. The recently announced $\sim 10 M_{E}$ planet CoRoT-7 b orbits a $\sim 0.75 M_{S}$ star and hence has a large geometric transit probability, but the transit depth was very low, requiring space-based observations to detect it.

Lower mass planets undergo less tidal heating and block less light, i.e., the yellow strips in Figure 1 move to the left, and the horizontal red lines move down. The geometric transit probability scales as the sum of the stellar and planetary radii (which scales with mass). But since terrestrial planet radii are much smaller than stellar radii, the diagonal red lines change negligibly with planetary mass. The IHZ is independent of the planetary mass and radius (as long as the planet is massive enough to retain an atmosphere).

Next we consider the ITHZ for a range of $M_{*}, M_{p}, a$, and $e$. Figure 2 shows the two $\mathrm{HZ}$ boundaries for 1 and $10 M_{E}$ planets (columns) orbiting 0.15 and $0.25 M_{S}$ stars (rows) as a function of $a$ and $e$. The color scheme is the same as in Figure 1.

As stellar mass increases, both the IHZ and THZ move to larger $a$, and the two HZs move more or less together. However, as planetary mass increases, the THZ may sweep through the IHZ, everything else held constant. The IHZ moves to larger $a$ at larger $e$ because the orbit-averaged flux increases with $e$

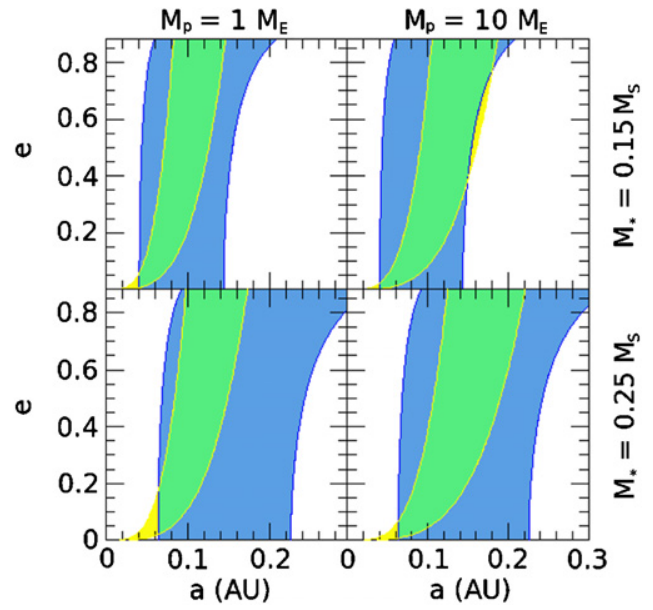

Figure 2. Habitability boundaries for a $1 M_{E}$ planet (left column) and $10 M_{E}$ planet (right column) orbiting a $0.15 M_{S}$ star (top row) and $0.25 M_{S}$ star (bottom row). This figure uses the same color scheme as Figure 1.

(Williams \& Pollard 2002). The tidal heating limits move to larger $a$ with larger $e$ because larger eccentricity leads to more heating.

We can apply these concepts to GJ 581 d (Mayor et al. 2009), whose mass and orbit were recently revised to $M_{p}=7.09 M_{E}$, $a=0.2184 \mathrm{AU}$, and $e=0.38$. These parameters predict the planet is in the Selsis et al. (2007) IHZ. However, using a stellar mass of $0.31 M_{S}$ (Udry et al. 2007), the tidal heat flux is 0.01 $\mathrm{W} \mathrm{m}{ }^{-2}=0.25 h_{\min }$. Therefore, our analysis excludes this planet from the ITHZ. However, if the planet has at least $0.03 \mathrm{~W} \mathrm{~m}^{-2}$ of radiogenic heating, it could still be habitable.

\section{DISCUSSION AND CONCLUSIONS}

We have shown that consideration of tidal heating and stellar flux are crucial when assessing planetary habitability. Tidal heating and detectability scale with stellar and planetary masses, but decrease with increasing $a$. Hence many planets found in the IHZ may in fact be uninhabitable due to intense global volcanism and rapid resurfacing. Consideration of tidal heating provides an important refinement in the definition of an HZ.

We have applied our model to the planet GJ $581 \mathrm{~d}$ (Mayor et al. 2009). This planet, although in the IHZ, does not reside in the THZ. However, this planet may still possess plate tectonics through radiogenic heating. At this point we cannot conclude that GJ $581 \mathrm{~d}$ is habitable. We encourage future work that examines the radiogenic heating of this planet.

We have assumed that tidal heating can drive plate tectonics, including subduction. For this possibility to be true, heating has to occur deep enough inside the planet to drive mantle convection. No models or observations of tidally driven subduction have been made. Such geophysical modeling would be illuminating and could necessitate a revision of the ideas presented here.

Our choice of $h_{\min }$, though rooted in observations in the solar system, could be inappropriate in some cases. The process of plate tectonics is studied by a large and active group of geophysicists, and as new results come to light, we may need to change our working definition of $h_{\min }$. Similarly, our choice for $h_{\max }$ may require revision. Planets do not recycle their lithosphere uniformly; "cool spots" could persist for long times and be oases for biogenesis. Furthermore, planets with unusual compositions or surface features may be able to maintain a 
biosphere even with such large heating. Future geophysical and biological modeling could elucidate the true value of $h_{\max }$.

Although we have generally focused on tidal heating, any heating with the potential to drive plate tectonics may stabilize climates. Therefore, the ITHZ is really a subset of a broader "insolation-geophysical HZ." It may be that very few planets have $h<h_{\text {min }}$ because radiogenic heating is generally larger than $h_{\min }$ (but only for a finite amount of time). However, the distribution of radiogenic isotopes in the galaxy remains unknown. Although the origin of short-lived radionuclides such as ${ }^{26} \mathrm{Al}$ in the solar system is not fully understood, models suggest that there should exist a dispersion of several orders of magnitude in their protoplanetary disks, and most planetary systems should actually contain less ${ }^{26} \mathrm{Al}$ than the solar system (Gaidos et al. 2009; see also Gounelle et al. 2009). If abundances of $\mathrm{K}$ and $\mathrm{U}$ (the main sources of internal heat on the Earth) also have a large dispersion, then many exoplanets may not support radiogenically driven plate tectonics. For such planets, tidal heating may be the only available energy source (Williams et al. 1997).

The orbital history of a planet, as well as where it is now, may be of critical importance when assessing habitability. Most planets inside 0.2 AU have orbits nearly circularized by tides from earlier eccentric orbits (Jackson et al. 2008c). As $e$ dropped (over billions of years), so did $a$. In general, $e$ decays more quickly than $a$ (planets tend to move vertically downward in Figure 2), so a planet can form interior to the ITHZ on a high-e orbit which is then circularized by tides into the ITHZ. If we detect a planet with such a history, it is probably not habitable. The reason is that large terrestrial planets, e.g., $\sim 10 M_{E}$, will tend to hold onto their atmospheres, and hence may be dominated by outgassed species. Thus, a planet in the ITHZ today could be uninhabitable due to a volatile past. Spectral analyses of transiting planets could reveal evidence of extreme volcanism, even though it is not active today. We encourage atmospheric modelers to consider the evolution of such a planet.

As tides modify the orbit, the eccentricity may eventually reach zero, and Equation (1) predicts no heat flux. Without radiogenic heating, the planet would become uninhabitable. However, other planets in the system may perturb the orbit and maintain a nonzero eccentricity (Mardling 2007), such as in the Galilean satellite system. Models of the formation of large terrestrial planets in small orbits suggest such planets may be likely (Raymond et al. 2008). Therefore, despite the persistent damping of $e$ by tides, some planets may reside in the ITHZ for a very long time.

As discussed in Section 1, tidal effects are but one of the several exotic issues that could affect the habitability of planets orbiting $\mathrm{M}$ stars. For example, the stripping of atmospheres due to massive stellar winds (ablation) is also likely a function of $M_{*}, M_{p}$, and $a$ (J. Zendejas et al. 2009, in preparation). In principle, detailed numerical modeling of these phenomena could identify an "ablation HZ," which could be compared to the ITHZ presented here. The identification of an "InsolationTidal-Ablation HZ" may guide the wide array of resources that are coming online to detect terrestrial mass planets. Other refinements to the limits of habitability incorporating other stellar and planetary phenomena, such as obliquity (Williams \& Pollard 2003; Spiegel et al. 2009), the presence of a large
Moon (Laskar et al. 1993; but see also Barnes \& O’Brien 2002), or the presence of water on the planet (Kuchner 2004; Raymond et al. 2007a), could be just as useful as the consideration of ablation.

R.B. and S.N.R. acknowledge funding from NASA Astrobiology Institute's Virtual Planetary Laboratory lead team, supported by NASA under Cooperative Agreement NNH05ZDA001C. B.J. acknowledges support from NESSF. R.G. acknowledges support from NASA's Planetary Geology and Geophysics program, grant NNG05GH65G. We thank Victoria Meadows and Shawn Domagal-Goldman for enlightening discussions.

\section{REFERENCES}

Barnes, J. 2007, PASP, 119, 986

Barnes, R., Jackson, B., Raymond, S. N., West, A. A., \& Greenberg, R. 2009, ApJ, 695, 1006

Barnes, J., \& O’Brien, D. P. 2002, ApJ, 575, 1087

Barnes, R., Raymond, S. N., Jackson, B., \& Greenberg, R. 2008, AsBio, 8, 557 Blaney, D. L., Johnson, T. V., Matson, D. L., \& Veeder, G. J. 1995, Icarus, 113 , 220

Butler, R. P., et al. 2006, ApJ, 646, 505

Davies, G. 1999, Dynamic Earth (Cambridge: Cambridge Univ. Press)

Dickey, J. O., et al. 1994, Science 265, 482

Efroimsky, M., \& Lainey, V. 2007, J. Geophys. Res., 112, E12003

Ferraz-Mello, S., Rodriguez, A., \& Hussman, H. 2008, CeMDA, 101, 171

Gaidos, E., Krot, A. N., Williams, J. P., \& Raymond, S. N. 2009, ApJ, 696, 1854 Goldreich, P., \& Soter, S. 1966, Icarus, 5, 375

Gounelle, M., Meibom, A., Hennebelle, P., \& Inutsuka, S. 2009, ApJ, 694, L1 Hut, P. 1981, A\&A, 99, 126

Ikoma, M., Emori, H., \& Nakazawa, K. 2001, ApJ, 553, 999

Jackson, B., Barnes, R., \& Greenberg, R. 2008a, MNRAS, 391, 237

Jackson, B., Greenberg, R., \& Barnes, R. 2008b, ApJ, 681, 1631

Jackson, B., Greenberg, R., \& Barnes, R. 2008c, ApJ, 678, 1396

Kasting, J. F., et al. 1993, Icarus, 101, 108

Kuchner, M. 2004, ApJ, 612, 1147

Lammer, H., et al. 2007, AsBio, 7, 185

Laskar, J., Joutel, F., \& Robutel, P. 1993, Nature, 361, 615

Levrard, B., et al. 2007, A\&A, 462, L5

Lissauer, J. J., et al. 2009, Icarus, 199, 338

Mardling, R. A. 2007, MNRAS, 382, 1768

Mardling, R. A., \& Lin, D. N. C. 2002, ApJ, 573, 829

Mayor, M., et al. 2009, A\&A, in press (arXiv:0906.2780)

McEwen, A. S., Keszthelyi, L. P., Lopes, R., Schenk, P. M., \& Spencer, J. R. 2004, in Jupiter: The planet, Satellites and Magnetosphere, ed. F. Bagenal, T. E. Dowling, \& W. B. McKinnon (Cambridge: Cambridge Univ. Press), 307

Nutzman, P., \& Charbonneau, D. 2008, PASP, 120, 317

Peale, S. J., Cassen, P., \& Reynolds, R. T. 1979, Science, 203, 892

Pollack, J. B., et al. 1996, Icarus, 194, 62

Raymond, S. N., Barnes, R., \& Mandell, A. M. 2008, MNRAS, 384, 663

Raymond, S. N., Quinn, T. R., \& Lunine, J. I. 2007a, AsBio, 7, 66

Raymond, S. N., Scalo, J., \& Meadows, V. 2007b, ApJ, 669, 606

Regenauer-Lieb, K., Yuen, D. A., \& Branlund, J. 2001, Science, 294, 578

Segura, A., et al. 2009, AsBio, in press

Selsis, F., et al. 2007, A\&A, 476, 137

Sotin, C., Grasset, O., \& Mocquet, A. 2007, Icarus, 191, 337

Spiegel, D. S., Menou, K., \& Scharf, C. A. 2009, ApJ, 691, 596

Udry, S., et al. 2007, A\&A, 469, L43

Walker, J. C. G., Hays, P. B., \& Kasting, J. F. 1981, J. Geophys. Res., 86, 9776

West, A. A., et al. 2004, AJ, 128, 426

Williams, D. M., Kasting, J. F., \& Wade, R. A. 1997, Nature, 385, 234

Williams, D. M., \& Pollard, D. 2002, Int. J. Astrobiol., 1, 61

Williams, D. M., \& Pollard, D. 2003, Int. J. Astrobiol., 2, 1

Winn, J. N., et al. 2008, ApJ, 683, 1076

Wisdom, J. 2008, Icarus, 193, 637

Yoder, C. F. 1995, in Global Earth Physics: A Handbook of Physical Constants, ed. T. Ahrens (Washington, DC: American Geophysical Union) 